Published in final edited form as:

Schizophr Res. 2013 May ; 146(0): 190-195. doi:10.1016/j.schres.2013.01.013.

\title{
Effects of switching from olanzapine, quetiapine, and risperidone to aripiprazole on 10-year coronary heart disease risk and metabolic syndrome status: Results from a randomized controlled trial
}

\author{
T. Scott Stroup ${ }^{a, b}$, Matthew J. Byerlyc, Henry A. Nasrallah ${ }^{d}$, Neepa Ray ${ }^{\mathrm{e}}$, Ahsan Y. Khan ${ }^{\dagger}$, J. \\ Steven Lambertig, Ira D. Glick ${ }^{h}$, Richard M. Steinbook', Joseph P. McEvoyj, and Robert M. \\ Hamer $^{\mathrm{e}}$ \\ aColumbia University College of Physicians and Surgeons \\ ${ }^{b}$ New York State Psychiatric Institute \\ 'University of Texas Southwestern \\ dUniversity of Cincinnati \\ eUniversity of North Carolina at Chapel Hill \\ fOklahoma University \\ gUniversity of Rochester \\ hStanford University \\ 'University of Miami \\ jDuke University Medical Center
}

\begin{abstract}
Purpose-This study examined the clinical significance of switching from olanzapine, quetiapine, or risperidone to aripiprazole by examining changes in predicted risk of cardiovascular disease (CVD) according to the Framingham Risk Score (FRS) and metabolic syndrome status.
\end{abstract}

(C) 2012 Elsevier B.V. All rights reserved.

Corresponding author: T. Scott Stroup, MD, MPH, Professor of Psychiatry, Columbia University College of Physicians and Surgeons, New York State Psychiatric Institute, Room 2073, Box 100, 1051 Riverside Drive, New York, NY 10032, stroups@ nyspi.columbia.edu, phone: 212-543-5676, fax: 212-543-5085.

Contributors

Drs. Stroup, McEvoy, and Hamer designed the study. Ms. Ray conducted the analyses. All the other authors conducted the study and contributed to the manuscript.

The study was conducted by the following investigators:

Lawrence Adler, Glen Burnie, MD; Farooq Amin, Atlanta, GA; Michael Barber, Houston, TX; Peter Buckley, Augusta, GA; Matthew Byerly, Dallas, TX; Jose Canive, Albuquerque, NM; Stanley Caroff, Philadelphia, PA; Cyril D'Souza, New Haven, CT; Ira Glick,

Palo Alto, CA; Daniel Mamah, St. Louis, MO; Don Goff, Boston, MA; Anita Kablinger, Shreveport, LA; Ahsan Kahn, Wichita, KS; Steven Lamberti, Rochester, NY; Jeffrey Lieberman, New York, NY; Theo Manschreck, Fall River, MA; Joseph McEvoy, Butner, NC; Alec Miller, San Antonio, TX; Del Miller, Iowa City, IA; James Rosenthal, Colson, CA; Henry Nasrallah, Cincinnati, OH; Stephen Olson, Minneapolis, MN; Jayendra Patel, Worcester, MA; Bruce Saltz, Boca Raton, FL; Richard Steinbook, Miami, FL; Scott Stroup, Chapel Hill, NC.

Publisher's Disclaimer: This is a PDF file of an unedited manuscript that has been accepted for publication. As a service to our customers we are providing this early version of the manuscript. The manuscript will undergo copyediting, typesetting, and review of the resulting proof before it is published in its final citable form. Please note that during the production process errors may be discovered which could affect the content, and all legal disclaimers that apply to the journal pertain. 
FRS estimates 10-year risk of "hard" coronary heart disease (CHD) outcomes (myocardial infarction and coronary death) while metabolic syndrome is associated with increased risk of CVD, stroke, and diabetes mellitus.

Method-Changes in FRS and metabolic syndrome status were compared between patients with BMI $\geq 27$ and non-HDL-C $\geq 130 \mathrm{mg} / \mathrm{dL}$ randomly assigned to stay on stable current treatment (olanzapine, quetiapine, or risperidone) or switch to treatment with aripiprazole with 24 weeks of follow-up. All study participants were enrolled in a behavioral program that promoted healthy diet and exercise.

Results-The pre-specified analyses included 89 switchers and 98 stayers who had post-baseline measurements needed to assess changes. Least squares mean estimates of 10-year CHD risk decreased more for the switch (from 7.0\% to 5.2\%) than the stay group (from 7.4\% to 6.4\%) $(\mathrm{p}=0.0429)$. The odds ratio for having metabolic syndrome (stay vs. switch) at the last observation was 1.748 (95\% CI 0.919, 3.324, $\mathrm{p}=0.0885)$.

Conclusion-Switching from olanzapine, quetiapine, or risperidone to aripiprazole was associated with larger reductions in predicted 10-year risk of CHD than the behavioral program alone. The advantage of switching on metabolic syndrome was not statistically significant. The benefits of switching must be balanced against its risks, which in this study included more discontinuations of the study treatment but no significant increase in symptoms or hospitalizations.

\section{Keywords}

Antipsychotics; Metabolic side effects; Randomized clinical trial

\section{Introduction}

The average life expectancy among individuals with schizophrenia in the U.S. is 61 years compared to 76 years for the general population, representing a 20\% reduced life expectancy (Hennekens, Hennekens et al. 2005). A major cause of premature death in schizophrenia is cardiovascular disease (CVD)(Osby, Correia et al. 2000; Hennekens, Hennekens et al. 2005; Bushe, Taylor et al. 2010). Many factors, including high rates of smoking, sedentary lifestyle, unhealthy diet, and a high prevalence of frequently untreated (Nasrallah, Meyer et al. 2006) hypertension and metabolic problems (obesity, dyslipidemia, and insulin resistance) contribute to the increased risk of CVD in patients with schizophrenia. In addition, antipsychotics are associated with varying degrees of adverse metabolic effects. For example, chlorpromazine, clozapine, olanzapine, quetiapine, and risperidone are associated with substantial weight gain and adverse metabolic effects, while aripiprazole, fluphenazine, haloperidol, ziprasidone and some newer antipsychotics have less prominent effects on these measures (Allison, Mentore et al. 1999; Newcomer 2005; Citrome 2011).

The Framingham Risk Score (FRS), derived from the Framingham Heart Study (Wilson, D'Agostino et al. 1998) is a clinically useful and widely used way to predict risk of coronary heart disease, including cardiac death, myocardial infarction, and angina. The FRS is a function of sex, age, HDL cholesterol, total cholesterol, systolic blood pressure, and presence of smoking. This risk score has been validated in several populations, and was used in the Clinical Antipsychotic Trials of Intervention Effectiveness (CATIE) schizophrenia study to demonstrate that individuals with schizophrenia had higher predicted CHD risk than matched controls from the general population (Goff, Sullivan et al. 2005). Additional analyses showed that the antipsychotic drugs studied in CATIE were associated with substantially different predicted 10-year risks of CHD (Daumit, Goff et al. 2008).

Metabolic syndrome is a clustering of several cardiovascular risk factors including central adiposity, hyperglycemia, dyslipidemia and hypertension (2002) and is another common 
approach to assess risk of cardiovascular disease-individuals with metabolic syndrome are at increased risk of CVD compared to those without metabolic syndrome. The intended use of the metabolic syndrome diagnosis is to identify individuals at risk of CVD and to initiate interventions to decrease the risk of premature mortality from cardiovascular disease. Metabolic syndrome, however, has been criticized as having an arbitrary number of criteria and unvalidated thresholds for meeting each of the criteria (Reaven 2005). In addition, metabolic syndrome is less accurate than the FRS in predicting CVD (Reaven 2011). Nevertheless, metabolic syndrome is widely understood as a marker of increased risk of CVD. Analyses from the CATIE trial demonstrated that the prevalence of metabolic syndrome in persons with chronic schizophrenia is much higher than matched controls from the general population (McEvoy, Meyer et al. 2005).

In this paper we examine the impact on the risk of cardiovascular disease, as measured by the FRS and metabolic syndrome, of switching from widely used antipsychotics that are known to be associated with adverse weight and metabolic effects (olanzapine, quetiapine, and risperidone) to treatment with aripiprazole, which is associated with a lower rate of such effects (2004; Newcomer 2005). The results of the primary and key secondary outcomes of the study, change in non-HDL cholesterol and efficacy failure, respectively, were previously published (Stroup, McEvoy et al. 2011).

\section{Method}

The Comparison of Antipsychotics for Metabolic Problems (CAMP) study is a multi-site randomized controlled clinical trial conducted between January 2007 and March 2010 at 27 clinical research centers affiliated with the Schizophrenia Trials Network (STN) in the U.S. (Stroup, McEvoy et al. 2011). Study participants were individuals with schizophrenia or schizoaffective disorder judged to be clinically stable on treatment with olanzapine, quetiapine, or risperidone, and who were at increased risk for cardiovascular disease as indicated by an elevated body-mass index (BMI) $\geq 27$ and a non-HDL-C $\geq 130 \mathrm{mg} / \mathrm{dl}$ (if non-HDL-C was 130 to 139 , then LDL cholesterol was required to be $\geq 100 \mathrm{mg} / \mathrm{dl}$ ). Patients with diabetes (fasting blood glucose $\geq 126 \mathrm{mg} / \mathrm{dl}$ or treated with insulin or an oral hypoglycemic drug) and severe dyslipidemias (non-HDL-C $>300 \mathrm{mg} / \mathrm{dl}$ or triglycerides $>$ $500 \mathrm{mg} / \mathrm{dl}$ ) were excluded because the study purpose was to examine whether switching could prevent such serious metabolic problems. Patients with a history of cardiovascular disease or cerebrovascular disease were excluded. Patients were treated with the qualifying drug for a minimum of three months and without dosage adjustments or the adjunctive use of any other antipsychotic medication for at least one month prior to enrollment. All participants provided written informed consent that was approved by the Institutional Review Board (IRB) of the participating sites. Patients were randomly assigned on a 1:1 basis to switch to aripiprazole or to stay on their current antipsychotic medication. Treatment assignment (stay vs. switch) was stratified by antipsychotic medication taken when entering the study (olanzapine, quetiapine, and risperidone). Individuals assigned to stay on the current antipsychotic treatment remained on the pre-study dosage with adjustments only as clinically indicated within allowed ranges. The allowed dosages were: olanzapine 5-20 mg daily, quetiapine 200-1200 mg daily, and risperidone 1-16 mg daily. Patients assigned to switch to aripiprazole began taking aripiprazole $5 \mathrm{mg}$ daily and continued their previous antipsychotic medication and dose for one week. Over the next 3 weeks the dosage of aripiprazole was gradually increased while the previous medication was tapered off. After 4 weeks the allowed dosage range for aripiprazole was 5-30 mg daily. Treatment was open-label but raters blinded to treatment assignment conducted the clinical ratings. Patients returned to the clinic for weekly visits during the first month of the treatment period and every four weeks after that. Laboratory assessments were conducted using fasting blood samples collected every four weeks. 
All participants received a manualized behavioral intervention, adapted from a group treatment (Brar, Ganguli et al. 2005; Ganguli 2007), that was aimed at improving exercise and diet habits. The behavioral intervention was provided in person at all post-baseline study visits. After the first four weeks, study personnel made a telephone call to reinforce the behavioral treatment lessons between each of the monthly visits.

\subsection{Primary outcome: definition and analyses}

The primary outcome was change in predicted 10-year CHD risk as measured by the Framingham Risk Score, calculated according to the formula developed by Wilson et al. (Wilson, D'Agostino et al. 1998). The score is a function of sex, age, HDL cholesterol, total cholesterol, systolic blood pressure, and presence of smoking.

The primary analysis compared the FRS change from baseline to 24 weeks between Stay and Switch groups for randomized participants who remained on study treatment for at least one month, which was the first time after baseline that outcome measures were collected. Longitudinal mixed effects linear models were used for the primary analysis. Model effects included main effects corresponding to incoming medication, baseline, visit week, treatment (stay vs. switch), as well as a treatment by time (i.e., visit) interaction term. As designed in our statistical analysis plan, our models included research site (with small sites pooled together) as a random effect, but in a small number of cases the actual effect of site was so small as to produce covariance parameters estimates close to zero, which caused computational instability and necessitated the removal of the site effect in those models. An unstructured variance-covariance matrix was used, and the primary contrast of interest was the comparison of least squares means in change from baseline between stay and switch at the 24-week visit. Other continuous outcome measures were analyzed using linear mixed models as described above for the primary analysis.

\subsection{Secondary outcome: definition and analyses}

Presence of metabolic syndrome was defined as having three or more of the following five components: elevated waist circumference defined as $\geq 40$ inches for males and $\geq 35$ inches for females; elevated fasting triglycerides, defined as $\geq 150 \mathrm{mg} / \mathrm{dL}$; reduced HDL cholesterol, defined as $<40 \mathrm{mg} / \mathrm{dL}$ for males and $<50 \mathrm{mg} / \mathrm{dL}$ for females; elevated blood pressure, defined as $\geq 130 / 85 \mathrm{~mm} \mathrm{Hg}$; and elevated fasting glucose, defined as $\geq 100 \mathrm{mg} / \mathrm{dL}$ (Alberti, Zimmet et al. 2005). Metabolic syndrome was analyzed at the last observed value for the 187 randomized participants who stayed on study treatment for at least one month. Cochran-Mantel-Haenszel tests stratified by incoming medication were used to compare treatment groups with respect to the proportion of participants experiencing metabolic syndrome. Logistic regression models were fit to compare the two treatment groups (stay vs. switch) with respect to metabolic syndrome, adjusting for incoming medication and metabolic syndrome at baseline. To determine maximum possible effect, change in metabolic syndrome status was also examined only for the 129 participants who completed the entire 24-week protocol on assigned treatment.

\section{Results}

A total of 215 patients were randomized; 109 were assigned to switch to aripiprazole and 106 to stay on the current antipsychotic. We focused our attention only on those study participants who could provide relevant data. The measures for the primary outcomes in this study were evaluated in 89 switchers and 98 stayers who completed at least one month of study participation and thus had at least one post-baseline visit at which the outcomes could be determined while on the assigned treatment (Table 1). Two participants never took study drug. Investigators attributed patient discontinuations of the protocol-specified treatment 
during the first month to the following reasons: lack of efficacy (stay $\mathrm{N}=0$, switch $\mathrm{N}=9$ ), unacceptable side effects (stay $\mathrm{N}=2$, switch $\mathrm{N}=3$ ), patient withdrew (stay $\mathrm{N}=2$, switch $\mathrm{N}=0$ ), lost to follow-up ( stay $\mathrm{N}=2$, switch $\mathrm{N}=5$ ), disallowed medicine (stay $\mathrm{N}=2$, switch $\mathrm{N}=1$ ) (Stroup, McEvoy et al. 2011). Table 1 depicts the baseline demographic and clinical characteristics of the 187 patients in these analyses. Mean daily doses for patients during the study were $16.9 \mathrm{mg}$ of aripiprazole, $18.0 \mathrm{mg}$ of olanzapine, $572.0 \mathrm{mg}$ of quetiapine, and 4.1 $\mathrm{mg}$ of risperidone.

\subsection{Change in 10-year coronary heart disease risk estimates}

Table 2 depicts covariate-adjusted changes in metabolic parameters over the course of the study for the groups that stayed on the current antipsychotic or switched to aripiprazole. At study entry the mean FRS for both groups combined was 0.073 , indicating a $7.3 \%$ risk of CHD over 10 years. Least squares mean estimates of 10-year CHD risk decreased more for the switch (from $7.0 \%$ to $5.2 \%$ ) than the stay group (from $7.4 \%$ to $6.4 \%)(\mathrm{p}=0.0429)$; thus the 10 -year risk reduction was $25 \%$ for switchers compared to $13.5 \%$ for stayers.

\subsection{Metabolic syndrome status}

Among the 187 patients who stayed on study medication at least one month, the prevalence of the metabolic syndrome at the last observation decreased for switchers (from 57\% to $45 \%$ ) and for stayers (from $66 \%$ to $60 \%$ ). The odds ratio for having metabolic syndrome (stay vs. switch) at the last observation was 1.748 (95\% CI 0.919, 3.324, p=0.0885). Results were similar among the 129 participants who completed the 24-week protocol on study treatment- the prevalence of metabolic syndrome decreased for switchers (from 55\% to $40.1 \%$ ) and for stayers (from $64.2 \%$ to $58.7 \%$ ).

\subsection{Results by gender}

We conducted additional post hoc analyses by gender (118 men and 69 women). For men the mean FRS at study entry was $8.6 \%$, while for women it was $5.1 \%$. (This compares to general population means of $7.0 \%$ for men and $4.2 \%$ for women calculated for a group of controls matched to participants in the CATIE study (Goff, Sullivan et al. 2005).) In the analysis including only men, the difference in reductions of predicted 10-year CHD risk between the switch $(2.3 \%)$ and stay groups $(0.8 \%)$ was not statistically significant $(\mathrm{p}=0.073)$. For women there was also no significant difference in the magnitude of reductions of predicted 10-year CHD risk between stayers (1.1\%) and switchers (0.8\%) $(\mathrm{p}=0.5161)$.

For men, the prevalence of metabolic syndrome decreased from $55.6 \%$ to $42.6 \%$ among switchers and from $65.6 \%$ to $60.9 \%$ for stayers. For women, the prevalence of metabolic syndrome decreased from $60.0 \%$ to $48.6 \%$ among switchers and from $67.6 \%$ to $58.8 \%$ for stayers.

\subsection{Results according to incoming drug strata}

Table 3 depicts covariate-adjusted changes in metabolic parameters over the course of the study according to the antipsychotic medication participants were taking when entering the study. For participants entering the study on olanzapine, those who switched to aripiprazole experienced a $26.3 \%$ reduction in FRS compared to a $12.5 \%$ reduction for stayers. For quetiapine, switchers experienced a $31.6 \%$ reduction in FRS compared to a $10.7 \%$ reduction for stayers. For risperidone, switchers experienced a $22.2 \%$ reduction in FRS compared to a $15.9 \%$ reduction for stayers. The odds ratios for having metabolic syndrome (stay vs. switch) at participants' last visit according to the incoming drug strata were as follows: 
olanzapine 1.035 (95\% CI 0.344, 3.110, $\mathrm{p}=0.9515)$, quetiapine 1.372 (95\% CI 0.364, 5.177, $\mathrm{p}=0.6407)$, and risperidone 3.309 ( $95 \%$ CI 1.187, 9.229, $\mathrm{p}=0.0222)$.

\section{Discussion}

We conducted a randomized controlled trial to test the effectiveness of switching antipsychotic medications to ameliorate adverse metabolic effects associated with cardiovascular disease. In a previous paper we reported that switching to aripiprazole was associated with decreased non-HDL cholesterol, weight, and triglycerides compared to staying on the current medication (olanzapine, quetiapine, or risperidone)(Stroup, McEvoy et al. 2011). In this paper, the second of two planned papers for the CAMP study, we have translated the study results into constructs that are useful and commonly used in clinical settings. The new analyses focusing on 10-year cardiovascular risk and metabolic syndrome status found that switching from treatment with olanzapine, quetiapine, or risperidone to treatment with aripiprazole resulted in decreased predicted risk of CHD as reflected by Framingham Risk Scores. The reduced prevalence of metabolic syndrome for switchers compared to stayers was not statistically significant.

The Framingham Risk Score is used clinically to identify individuals who can be targeted for educational efforts and for lifestyle and medical interventions. Reductions in predicted CHD risk are an indication of the benefits of an intervention. In this study, twenty-four weeks of switch to aripiprazole resulted in calculated risk reduction in predicted CHD events over 10 years from $7.0 \%$ to $5.2 \%$ (reduction of $25.7 \%$ ) for switchers while the stayers, who received the behavioral program alone, had a reduced risk of $13.5 \%$. To provide a comparison of the magnitude of this effect, a recent meta-analysis by the Cochrane group found that treatment durations of 2.4 to 5.2 years led to a $28 \%$ relative risk reduction in observed CHD events for statins (vs. placebo) for primary prevention of CHD (Taylor, Ward et al. 2011). It remains to be determined if large prospective trials powered for CHD events would show actual risk reduction similar to the estimated effect in the current study and whether the magnitude of impact for switching of antipsychotics can be enhanced through longer intervention periods. The number of participants in the CAMP study, while not large, was adequate to test the primary study hypothesis and to find statistically significant and clinically meaningful findings in the current analyses.

A strength of the current study is that it was specifically designed to examine factors associated with risk of cardiovascular disease. We note that the mean baseline FRS in this study was lower in this study than that in the CATIE schizophrenia trial (Goff, Sullivan et al. 2005) even though individuals volunteered to participate in this study because they wanted to reduce CVD risk. One likely reason is that the present study excluded individuals with diabetes and those with clinically severe dyslipidemias. While the CATIE study was meant to be as inclusive as possible, the goal of the CAMP study was to evaluate the effectiveness of switching to prevent the serious problems that were so prevalent in the CATIE study. Because participants in the CAMP study were at risk of cardiovascular disease and diabetes but did not yet have these conditions, results from this study best generalize to this commonly encountered group. The reduction in CHD risk found with antipsychotic switching in this study could be different in more inclusive populations found in routine care.

One limitation of the study is its coupling of the medication switch with a diet and exercise program. Because structured behavioral programs are not routinely offered in typical care settings, this may limit the generalizability of findings. In addition, the use of such a program in this study's control group and in conjunction with switching may have attenuated the differences in the groups compared to typical practice in which the likely 
options are switching alone compared to staying alone. In addition, because the patients in this study wanted to reduce their risk of CHD, the benefits of the behavioral intervention were likely greater in this motivated group than in general populations.

Because the long-term trajectory of metabolic changes is uncertain, another limitation is the study's relatively short intervention period (24 weeks). In this study, the benefits of switching to aripiprazole on serum triglycerides and non-HDL cholesterol were realized in the first month of treatment and then maintained, while weight reduction advantages continued to accrue over the study period (Stroup, McEvoy et al. 2011). Although behavioral programs may have long-term benefits for those who continue to participate, poor retention in such programs may limit their long-term impact (Holt, Pendlebury et al. 2010).

All study participants in the study received close monitoring to minimize the risk of clinical destabilization. Nine $(8.3 \%)$ switchers to aripiprazole and no $(0 \%)$ stayers discontinued protocol-specified treatment in the first month due to lack of efficacy, as judged by the investigator (Stroup, McEvoy et al. 2011). Over the entire 24 weeks, 18 (16.8\%) switchers stopped aripiprazole and three (2.8\%) stayers stopped study medication due to investigatorjudged lack of efficacy (Stroup, McEvoy et al. 2011). These judgments by investigators who were not blinded to treatment assignment are in sharp contrast to rates of protocol-specified efficacy failure, which required hospitalization or blinded ratings that found substantial worsening of psychopathology. By this more objective measure, rates of efficacy failure were similar for the switchers $(\mathrm{N}=22,20.6 \%)$ and stayers $(\mathrm{N}=18,17.0 \%)(\mathrm{p}=0.4782)$ (Stroup, McEvoy et al. 2011). In contrast, an open-label pilot study of 15 patients who tried switching to aripiprazole also reported a high rate (33\%) of stopping aripirazole due to "actual or fear of symptom worsening" (Kim, Ivanova et al. 2007). These results strongly suggest that blinding is important to avoid expectation biases in studies involving clinical endpoints. Aripiprazole, while clearly an efficacious treatment for schizophrenia (El-Sayeh and Morganti 2006), has been shown in a meta-analysis to be less efficacious than olanzapine (Leucht, Komossa et al. 2009). Close clinical monitoring is necessary during and after a switch to aripiprazole or any other antipsychotic medication.

Results according to incoming drug strata and by gender should be considered exploratory. For example, it is surprising that switches from risperidone but not from olanzapine or quetiapine were associated with reductions in metabolic syndrome prevalence. The nominal statistical significance for findings in these exploratory analyses may suggest real effects or chance.

In conclusion, switching from olanzapine, quetiapine, or risperidone to aripiprazole was associated with reduced predicted risk of CHD. Both the 25\% reduction in FRS associated with switching to aripiprazole together with the behavioral program and the $13.5 \%$ reduction associated with the behavioral program alone are clinically meaningful. Switches to other antipsychotic medications that have favorable metabolic profiles are other options.

Clinicians may also consider recommending metformin, statins, diet and exercise programs, and smoking cessation programs as alternative approaches that can be considered alone or in conjunction with switching medications. One advantage of the switching strategy, compared to the use of adjunctive medications, is that it avoids additional medications, potential drug interactions and additional costs. However, a switch strategy may not always be effective (Stroup, McEvoy et al. 2011). Although switching in this study was not associated with significantly more efficacy failures or hospitalizations, the process of changing antipsychotic medications to ameliorate metabolic adverse effects can be unpredictable and requires close clinical monitoring. The effects on reductions of CHD risk associated with switching to aripiprazole over periods longer than 24 weeks are not clear and should be examined, as 
should the possible benefits of switching to other antipsychotics with similar weight and metabolic effects. The benefit of the behavioral program on cardiovascular risk over 24 weeks in this study suggests that this important intervention also warrants further long-term study. Other efforts to reduce the risks of cardiovascular disease, such as smoking cessation, are also warranted.

\section{Acknowledgments}

The study was funded by a grant from the Foundation for the National Institutes of Health (FNIH) and by a contract from the National Institute of Mental Health (N01 MH900001). Bristol Myers Squibb (BMS) provided aripiprazole and funds to FNIH in support of the study. FNIH, NIMH, and BMS were not involved in the conduct of the study, data analysis, or the preparation of this manuscript.

\section{References}

Third Report of the National Cholesterol Education Program (NCEP) Expert Panel on Detection, Evaluation, and Treatment of High Blood Cholesterol in Adults (Adult Treatment Panel III) final report. Circulation. 106(25):3143-3421.

Consensus development conference on antipsychotic drugs and obesity and diabetes. J Clin Psychiatry. 65(2):267-272.

Alberti KG, Zimmet P, et al. The metabolic syndrome--a new worldwide definition. Lancet. 2005; 366(9491):1059-1062. [PubMed: 16182882]

Allison DB, Mentore JL, et al. Antipsychotic-induced weight gain: a comprehensive research synthesis. The American journal of psychiatry. 1999; 156(11):1686-1696. [PubMed: 10553730]

Brar JS, Ganguli R, et al. Effects of behavioral therapy on weight loss in overweight and obese patients with schizophrenia or schizoaffective disorder. J Clin Psychiatry. 2005; 66(2):205-212. [PubMed: 15705006]

Bushe CJ, Taylor M, et al. Mortality in schizophrenia: a measurable clinical endpoint. Journal of psychopharmacology. 2010; 24(4 Suppl):17-25. [PubMed: 20923917]

Citrome L. Iloperidone, asenapine, and lurasidone: a brief overview of 3 new second-generation antipsychotics. Postgraduate medicine. 2011; 123(2):153-162. [PubMed: 21474903]

Daumit GL, Goff DC, et al. Antipsychotic effects on estimated 10-year coronary heart disease risk in the CATIE schizophrenia study. Schizophrenia research. 2008; 105(1-3):175-187. [PubMed: 18775645]

El-Sayeh HG, Morganti C. Aripiprazole for schizophrenia. Cochrane database of systematic reviews. 2006; (2):CD004578.

Ganguli R. Behavioral therapy for weight loss in patients with schizophrenia. J Clin Psychiatry. 2007; 68(Suppl 4):19-25. [PubMed: 17539696]

Goff DC, Sullivan LM, et al. A comparison of ten-year cardiac risk estimates in schizophrenia patients from the CATIE study and matched controls. Schizophrenia research. 2005; 80(1):45-53. [PubMed: 16198088]

Hennekens $\mathrm{CH}$, Hennekens AR, et al. Schizophrenia and increased risks of cardiovascular disease. American heart journal. 2005; 150(6):1115-1121. [PubMed: 16338246]

Holt RI, Pendlebury J, et al. Intentional weight loss in overweight and obese patients with severe mental illness: 8-year experience of a behavioral treatment program. The Journal of clinical psychiatry. 2010; 71(6):800-805. [PubMed: 20361917]

Kim SH, Ivanova O, et al. Metabolic impact of switching antipsychotic therapy to aripiprazole after weight gain: a pilot study. Journal of clinical psychopharmacology. 2007; 27(4):365-368. [PubMed: 17632220]

Leucht S, Komossa K, et al. A meta-analysis of head-to-head comparisons of second-generation antipsychotics in the treatment of schizophrenia. Am J Psychiatry. 2009; 166(2):152-163. [PubMed: 19015230]

McEvoy JP, Meyer JM, et al. Prevalence of the metabolic syndrome in patients with schizophrenia: baseline results from the Clinical Antipsychotic Trials of Intervention Effectiveness (CATIE) 
schizophrenia trial and comparison with national estimates from NHANES III. Schizophr Res. 2005; 80(1):19-32. [PubMed: 16137860]

Nasrallah HA, Meyer JM, et al. Low rates of treatment for hypertension, dyslipidemia and diabetes in schizophrenia: data from the CATIE schizophrenia trial sample at baseline. Schizophr Res. 2006; 86(1-3):15-22. [PubMed: 16884895]

Newcomer JW. Second-generation (atypical) antipsychotics and metabolic effects: a comprehensive literature review. CNS Drugs. 2005; 19(Suppl 1):1-93. [PubMed: 15998156]

Osby U, Correia N, et al. Mortality and causes of death in schizophrenia in Stockholm County, Sweden. Schizophrenia Research. 2000; 45(1-2):21-28. [PubMed: 10978869]

Reaven GM. The metabolic syndrome: requiescat in pace. Clinical chemistry. 2005; 51(6):931-938. [PubMed: 15746300]

Reaven GM. The metabolic syndrome: time to get off the merry-go-round? Journal of internal medicine. 2011; 269(2):127-136. [PubMed: 21129047]

Stroup TS, McEvoy JP, et al. A Randomized Trial Examining the Effectiveness of Switching From Olanzapine, Quetiapine, or Risperidone to Aripiprazole to Reduce Metabolic Risk: Comparison of Antipsychotics for Metabolic Problems (CAMP). The American journal of psychiatry. 2011; 168(9):947-956. [PubMed: 21768610]

Taylor F, Ward K, et al. Statins for the primary prevention of cardiovascular disease. Cochrane database of systematic reviews. 2011; (1):CD004816.

Wilson PW, D'Agostino RB, et al. Prediction of coronary heart disease using risk factor categories. Circulation. 1998; 97(18):1837-1847. [PubMed: 9603539] 
Table 1

Baseline Demographic and Clinical Characteristics of Efficacy Evaluable Population

\begin{tabular}{|c|c|c|c|}
\hline & Total $(\mathrm{N}=187)$ & Switch $(\mathrm{N}=89)$ & Stay $(\mathrm{N}=98)$ \\
\hline \multicolumn{4}{|l|}{ Demographic characteristics } \\
\hline Age - yr & $41 \pm 11.1$ & $40 \pm 11.8$ & $42 \pm 10.5$ \\
\hline \multicolumn{4}{|l|}{ Sex - no. $(\%)$} \\
\hline Male & $118(63.1)$ & $54(60.7)$ & $64(65.3)$ \\
\hline Female & $69(36.9)$ & $35(39.3)$ & $34(34.7)$ \\
\hline \multicolumn{4}{|l|}{ Race - no. $(\%)$} \\
\hline White & $106(56.7)$ & $52(58.4)$ & $54(55.1)$ \\
\hline Non-White & $80(42.8)$ & $37(41.6)$ & $43(43.9)$ \\
\hline Spanish, Hispanic or Latino ethnicity - no. (\%) & $34(18.2)$ & $15(16.9)$ & $19(19.4)$ \\
\hline Education - yr & $12 \pm 2.5$ & $12 \pm 2.3$ & $12 \pm 2.7$ \\
\hline High school education or above - no. (\%) & $144(77.0)$ & $67(75.3)$ & 77 (78.6) \\
\hline \multicolumn{4}{|l|}{ Marital Status - no. (\%) } \\
\hline Married & $15(8.0)$ & $8(9.0)$ & $7(7.1)$ \\
\hline Previously Married & $59(31.6)$ & $31(34.8)$ & $28(28.6)$ \\
\hline Never Married & $113(60.4)$ & $50(56.2)$ & $63(64.3)$ \\
\hline \multicolumn{4}{|l|}{ Living Situation - no. (\%) } \\
\hline Independent/non-structured & $157(84.0)$ & $74(83.1)$ & $83(84.7)$ \\
\hline Other & $30(16.0)$ & $15(16.9)$ & $15(15.3)$ \\
\hline \multicolumn{4}{|l|}{ Incoming antipsychotic medication -no. (\%) } \\
\hline Olanzapine & $64(34.2)$ & $29(32.6)$ & $35(35.7)$ \\
\hline Quetiapine & $51(27.3)$ & $23(25.8)$ & $28(28.6)$ \\
\hline Risperidone & $72(38.5)$ & $37(41.6)$ & $35(35.7)$ \\
\hline \multicolumn{4}{|l|}{ Smoking - no. (\%) } \\
\hline Yes & $104(55.6)$ & $50(56.2)$ & $54(55.1)$ \\
\hline No & $82(43.9)$ & $38(42.7)$ & $44(44.9)$ \\
\hline \multicolumn{4}{|l|}{ Clinical characteristics } \\
\hline Metabolic Syndrome - no. (\%) & $116(62.0)$ & $51(57.3)$ & $65(66.3)$ \\
\hline
\end{tabular}




\begin{tabular}{lccc}
\hline & Total $(\mathbf{N}=\mathbf{1 8 7})$ & Switch $(\mathbf{N}=\mathbf{8 9})$ & Stay (N=98) \\
\hline Weight - kg & $104 \pm 21.1$ & $104 \pm 21.4$ & $103 \pm 21.0$ \\
\hline Fasting glucose - mg/dL & $96 \pm 11.5$ & $96 \pm 10.9$ & $97 \pm 12.0$ \\
\hline LDL Cholesterol - mg/dL & $140 \pm 28.4$ & $138 \pm 26.4$ & $142 \pm 30.1$ \\
\hline HDL Cholesterol - mg/dL & $44 \pm 11.5$ & $45 \pm 12.7$ & $43 \pm 10.1$ \\
\hline Triglycerides - mg/dL & $172 \pm 96.4$ & $163 \pm 90.7$ & $180 \pm 101.1$ \\
\hline TG/HDL ratio & $4.4 \pm 3.19$ & $4.2 \pm 3.08$ & $4.7 \pm 3.29$ \\
\hline Waist circumference (inches) & $44 \pm 5.7$ & $44 \pm 5.7$ & $44 \pm 5.8$ \\
\hline Systolic blood pressure (mmHg) & $128 \pm 15.2$ & $127 \pm 14.2$ & $130 \pm 15.9$ \\
\hline Diastolic blood pressure (mmHg) & $84 \pm 11.8$ & $83 \pm 11.7$ & $84 \pm 12.0$ \\
\hline Framingham risk score & $0.073 \pm 0.057$ & $0.070 \pm 0.056$ & $0.074 \pm 0.058$ \\
\hline
\end{tabular}


Table 2

Outcome Measures of Effectiveness in the Efficacy Evaluable Population - by treatment

\begin{tabular}{lllll}
\hline & Switch $(\mathbf{N}=\mathbf{8 9})$ & Stay $(\mathbf{N}=98)$ & Difference (95\% CI) & P value* \\
\hline Primary Outcome & & & & \\
\hline Change in Framingham Risk Score & $-0.018 \pm 0.0030$ & $-0.010 \pm 0.0026$ & $0.008(0.000,0.016)$ & $0.0429^{\#}$ \\
\hline Other Secondary Outcomes & & & & \\
\hline Metabolic Outcomes & $-3.6 \pm 0.48$ & $-0.7 \pm 0.44$ & $29(1.6,4.2)$ & $<.0001^{\#}$ \\
\hline Change in weight (kg) & $-15.4 \pm 27.6$ & $-12.5 \pm 2.54$ & $2.9(-3.9,9.6)$ & 0.4003 \\
\hline Change in LDL Cholesterol (mg/dL) & $0.6 \pm 0.76$ & $-0.1 \pm 0.68$ & $-0.7(-2.7,1.3)$ & $0.4693^{\#}$ \\
\hline Change in HDL Cholesterol (mg/dL) & $-25.7 \pm 8.10$ & $7.0 \pm 7.20$ & $32.7(12.1,53.4)$ & 0.0020 \\
\hline Change in Triglycerides (mg/dL) & $-0.8 \pm 0.25$ & $0.2 \pm 0.22$ & $1.0(0.3,1.6)$ & $0.0029^{\#}$ \\
\hline Change in TG/HDL ratio & $0.5 \pm 1.43$ & $4.0 \pm 1.25$ & $3.5(-0.2,7.1)$ & 0.0607 \\
\hline Change in fasting glucose (mg/dL) & $-1.4 \pm 0.25$ & $-0.5 \pm 0.22$ & $0.9(0.2,1.5)$ & $0.0075^{\#}$ \\
\hline Change in waist circumference (inches) & $-3.4 \pm 1.39$ & $-1.0 \pm 1.23$ & $23(-1.0,5.7)$ & 0.1753 \\
\hline Change in systolic bood pressure (mmHg) & $-3.2 \pm 1.18$ & $-1.8 \pm 1.07$ & $1.4(-1.2,4.0)$ & 0.2937 \\
\hline Change in diastolic blood pressure (mmHg)
\end{tabular}




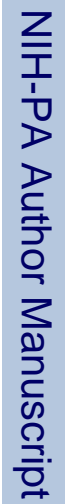

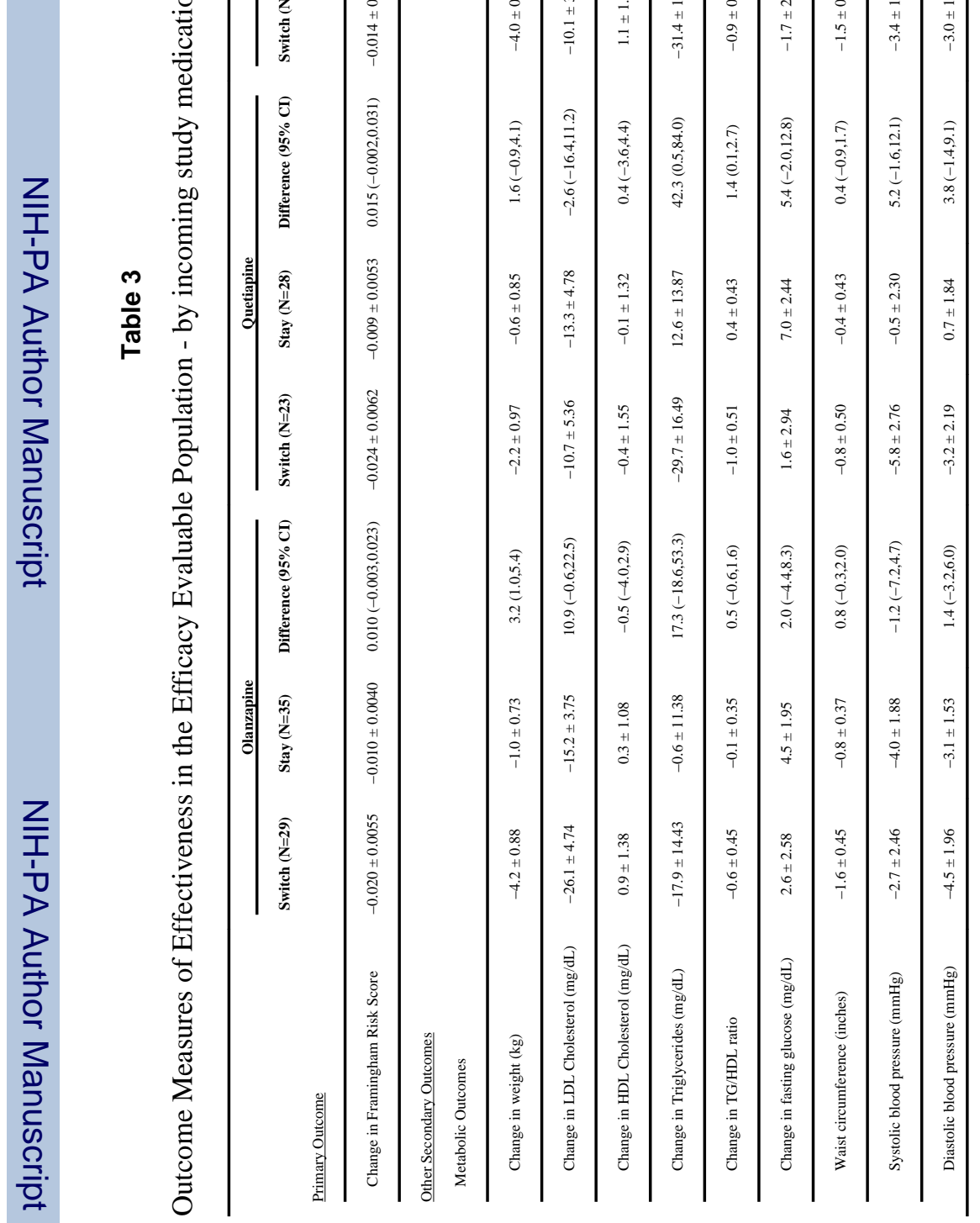

